\title{
Binary phase-controlled multi-beam- switching antenna array for reconfigurable 5G applications
}

\author{
Vedaprabhu Basavarajappa ${ }^{1,2^{*}}$ (D), Beatriz Bedia Exposito ${ }^{1}$, Lorena Cabria ${ }^{1}$ and Jose Basterrechea ${ }^{2}$
}

\begin{abstract}
The paper presents a two-phase state based multi-beam-switching scheme implemented on a customdesigned $4 \times 4$ antenna array operating with a bandwidth of $1.5 \mathrm{GHz}$ around $14 \mathrm{GHz}$. The antenna array and the beam-switching scheme have been experimentally validated. A phasing network designed to produce two phase states is used to experimentally validate the beam-switching and five beam states are presented, though this can be extended to other configurations using the split beam as a building block to construct multiple beams. The antenna can find potential use in multi-user millimetre-wave massive MIMO scenarios which require simultaneous multiple beams along selective directions.
\end{abstract}

Keywords: Multi-beam antenna, Beam-switching, Massive MIMO, Millimetre wave, 5G

\section{Introduction}

In the quest for antennas that can support high data rates demanded by 5 th generation (5G) communications, an interdependent string of requirements that characterize these antennas has recently been developed. Most prominent among these are the requirements of low-profile and high-radiation efficiencies [1] for the operation of the antennas in millimetre wave. In addition, there is a need for wideband, multi-frequency antennas with simpler fabrication and ease of deployment. At millimetre wave, it is well known that the antennas require high gain to combat the high path loss at those frequencies. Endfire antennas with directors and aided parasitics help in achieving these high gains. The next plausible step in the design of these antennas is the incorporation of high gain multi-beam capabilities. These techniques are generally based on reflectors, lenses

\footnotetext{
* Correspondence: veda@ieee.org

${ }^{1}$ Department of Antennas, TTI Norte, Parque Científico y Tecnológico de Cantabria, C/ Albert Einstein n 14, 39011 Santander, Spain

2Departamento de Ingeniería de Comunicaciones, Universidad de Cantabria, Av. de los Castros s/n, 39005 Santander, Spain
}

or beamforming circuits [2]. Additional ways to realize multi-beam operation recently have centred around reconfigurable techniques, finite large arrays, beam tilting and beam switching [2]. This paper focuses on the proposal and validation of a simple beam-switching technique based only on two-phase states using a $4 \times 4$ antenna array. The proposal uses a new radiating element initially introduced in [3], although other appropriate radiating elements can be used for the purpose.

A brief survey of recent antenna array design techniques to realize multi-beam is discussed next. The paper in [4] introduces a way to beam-switch along the azimuthal plane along $360^{\circ}$ without the use of conventional butler matrices, phase feeding networks or ESPAR-based strategies. It uses quasi-lumped couplers (QLCs) which are a pair of parallel transmission lines separated by two tunable varactors giving rise to a four-port network. The ratio of the power between the input port and the output port of each QLC can be controlled by varying the capacitance of the varactor. A circular cascaded network of the QLCs is used to attain a round-robin way to circulate power to attain operations in both single and multi-mode over Yagi antennas. However, to switch between multiple elements along the circle would 


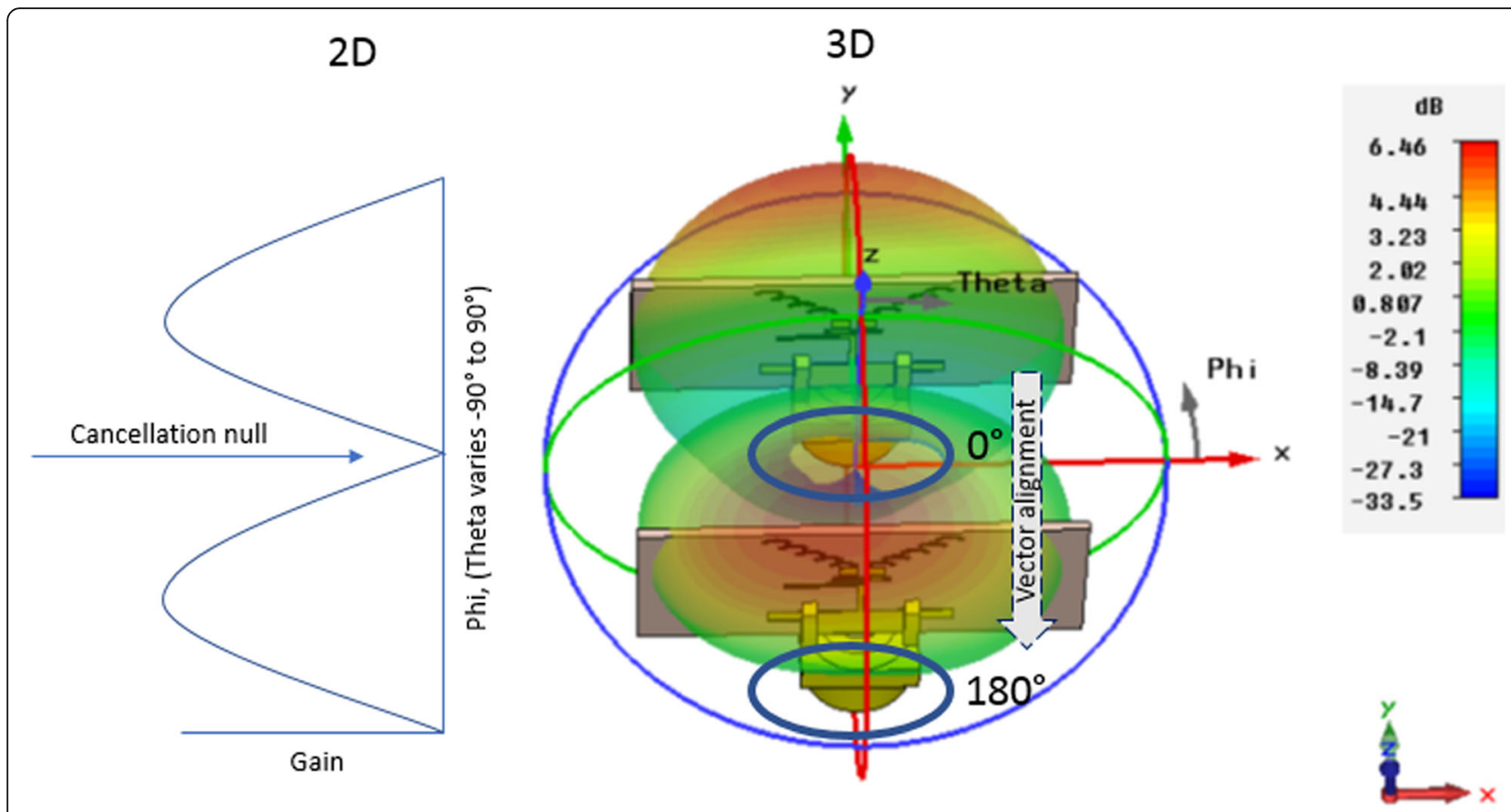

Fig. 1 Building block of the multi-beam scheme

mean the design of multiple multi-layered reconfigurable rat-race couplers which is a limitation of this proposal. The scalability of the design to higher frequencies would necessitate the consideration of appropriate substrate material considerations and tangent losses, which in turn would limit the radiation efficiencies. Guan et al. [5] present a way to attain multi-beam radiation using a $3 \times 3$ antenna array of probe-fed patch antennas without a beamforming network. The probe-fed patch antennas are coupled to each other using four microstrip lines that act as a substitute for the feeding network. Up to nine beams can be obtained with a gain around $10 \mathrm{dBi}$. The paper in [6] investigates the impact of mutual coupling and edge effects on the gain variation along large arrays. Two arrays made of dipoles and patch elements are compared. It is proposed that due to the omnidirectional pattern of the dipoles, the gain variation is more due to the increased mutual coupling between the elements and an increased observed deviation is seen in the embedded element gain. On the contrary, in the directional patch elements, the coupling is much lower. The arrays are composed of 32 elements each and their performance is evaluated for edge effects in a finite configuration. The paper in [7] proposes a way to switch between five beams and tilt each of the beams in the plane perpendicular to the comb-line

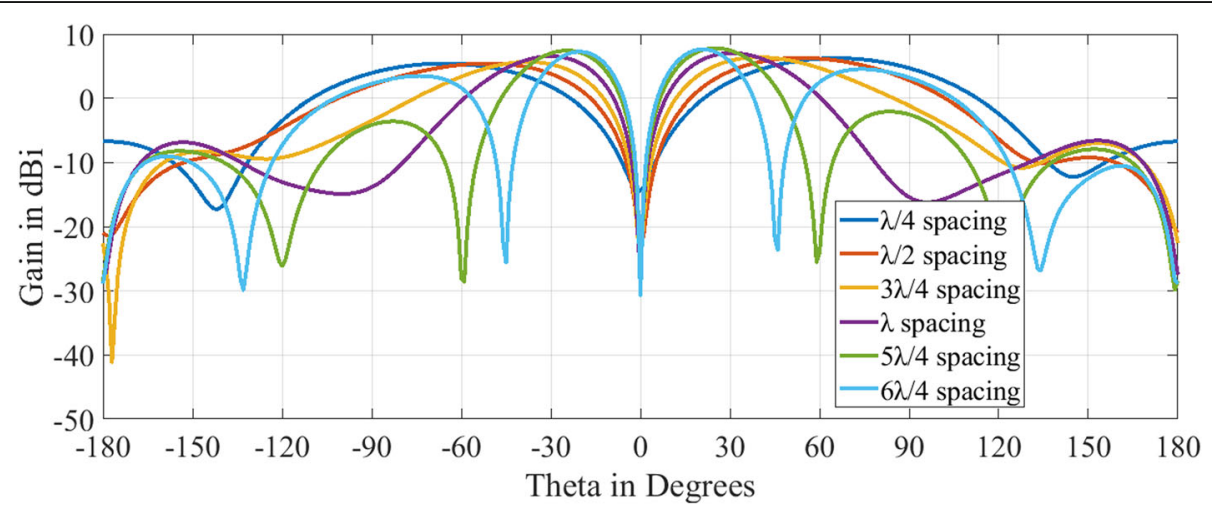

Fig. 2 Interelement spacing dependent phasing-enforced, beam pointing for two element array 


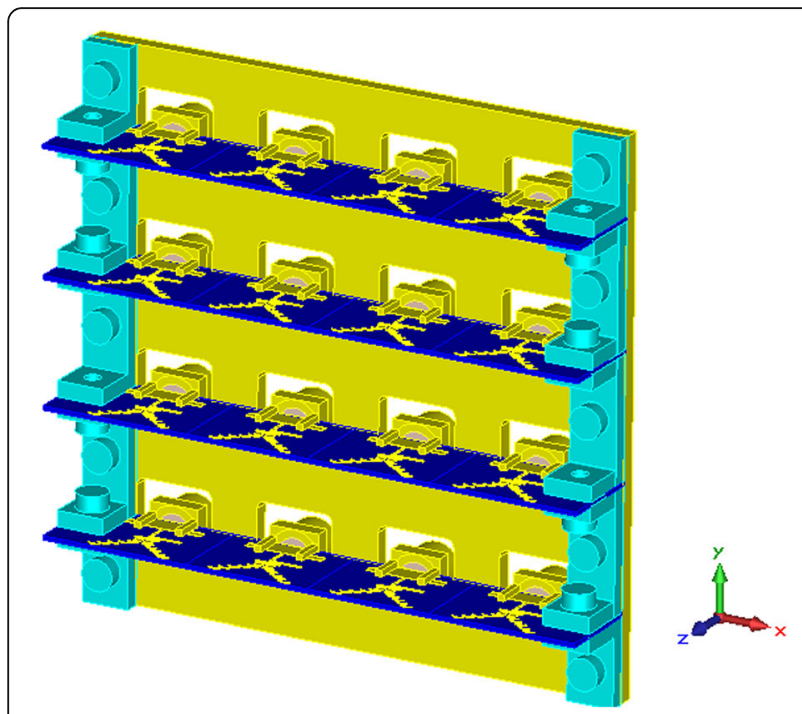

Fig. 3 Simulation model of the $4 \times 4$ array with supporting structure

feed. Five parallel comb-line feed networks consisting of three lines each are fed by a 3-way power divider using a RF switch. There are five such RF switches each connecting a triplet of comb lines. The phasing provided to the comb lines is controlled by changing the length of the transmission lines between the feeding point and the radiating element. The antenna provides tilt in the elevation plane.

A comparison of the proposed antenna array and its operation to the above-related works is presented next. In comparison to the paper in [4], the proposed beam-switching scheme operates in the radiating half-plane rather than in the azimuth $360^{\circ}$ plane. It also operates at a frequency of $14 \mathrm{GHz}$ as compared to $1 \mathrm{GHz}$ in the QLC design and does not employ multi-layered rat-race couplers for multimode beam-switching operation. In contrast to the paper in [5], which uses beam steering without a feeding network, the proposed antenna array uses a beam-switching scheme with a twovector state phase feeding network. Multiple simultaneous beams of up to four at a time can be obtained using the proposed feeding scheme. The operational frequency is of the same order. The paper in [6] states that in the case of the directional patch array, there is a lower gain variation between elements at angles closer to the direction normal to the array; similar observations have been made and verified experimentally in the proposed $4 \times 4$ antenna array. The gain along the boresight is similar with no much variation, although for oblique directions to the normal, there is a slight drop in gain. The paper in [6] also concludes that more directive patch elements are a better choice for massive MIMO. In comparison to the paper in [7], the proposed $4 \times 4$ antenna array provides multi-beams using a two-vector state beamswitching scheme and operates at $14 \mathrm{GHz}$. The required phasing is provided by the transmission line phasing network specifically designed for validation purposes.

After this introductory section that presents a survey of the state-of-art in multi-beam antennas and attempts to relate the proposed antenna in the contextual arena with a discussion on the compared features of the different methodologies, the rest of the paper is organized as follows: Section 2 provides the beam-switching characteristics of the antenna array and presents the novel two-vector beamswitching method to generate simultaneous highgain multiple beams. Section 3 details the measurements and provides a discussion on the multi-beam pattern and the gain measured. Finally, Section 4 concludes.

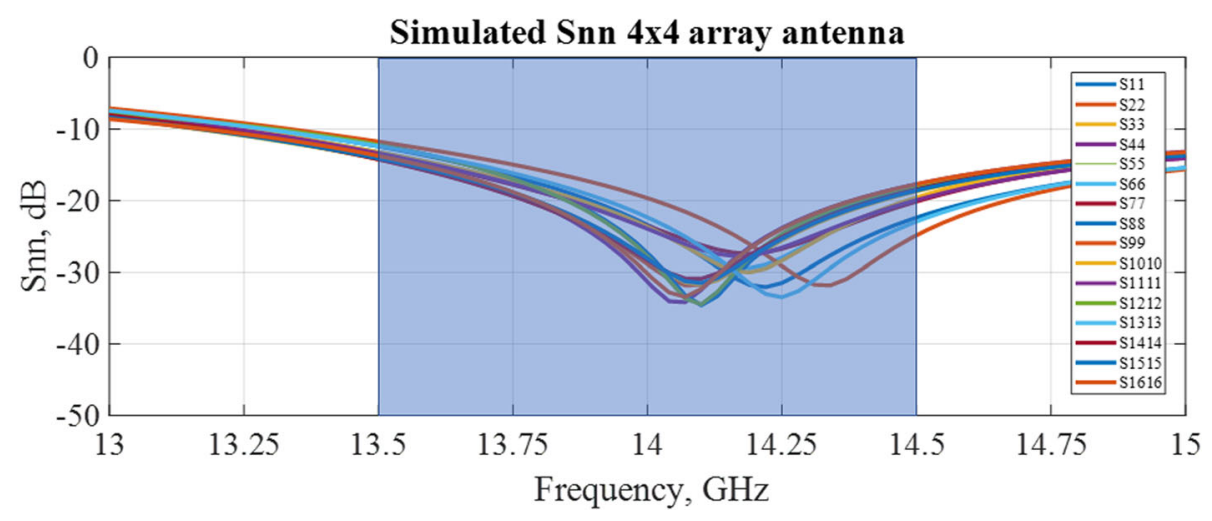

Fig. 4 Simulated return loss (Snn) of the $4 \times 4$ array antenna 


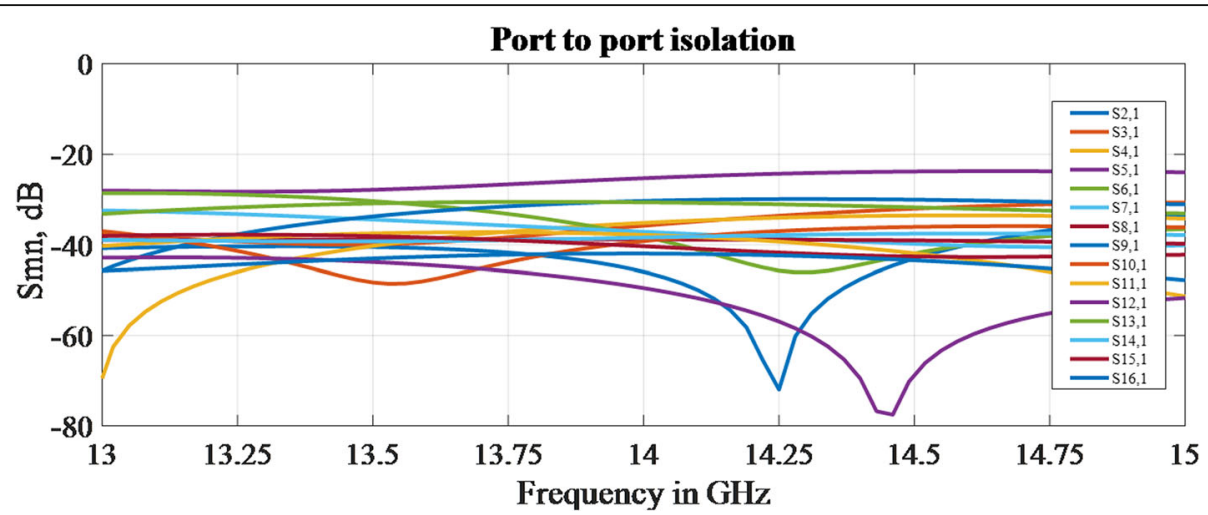

Fig. 5 Port-to-port isolation with respect to port 1

\section{Antenna array design and beam-switching concept}

\subsection{Fundamental beam unit}

To realize beam steering or beam switching, the general procedure followed over an antenna array is to primarily control the way the amplitude and phases are fed into the elements of the antenna array [8-10]. Other factors such as the antenna array inter-element spacing and the individual element radiation pattern also play a crucial role. In course of achieving the desired radiation pattern, which may at times be a single lobe that is steered to oblique angles or multi-beam generation, pattern synthesis methods can be used [11-13]. This often involves weighting the amplitudes and phases in predefined ways such as the Dolph-Chebyshev or the binomial distribution where the chosen amplitudes and phases affect the main lobe direction and the SLL. These and other such beam-steering methods are promising owing to the freedom of beam steerability they introduce into the structure. An additional requirement of these methods is that the distinct phases and amplitude must be actively fed into each element of the antenna array which can often be expensive owing to the use of wideband/multi-band phase shifters and their corresponding active dynamic control. To realize almost a similar functionality using a passive phasing network, it is necessary to have a beam-switching scheme that minimizes the required phase states, so that multi-beam operation can be attained with simpler networks. Such a scheme is proposed in this paper that can generate multiple patterns in certain directions using minimal phase reconfiguration of the feeding network. It uses a unique way to control the direction of the patterns using two phases or binary phase control. This binary phase control of $0^{\circ}$ and $180^{\circ}$ when applied in conjunction with a variation of the interelement spacing can produce unique multi-beam patterns along certain directions. The feeding network can be

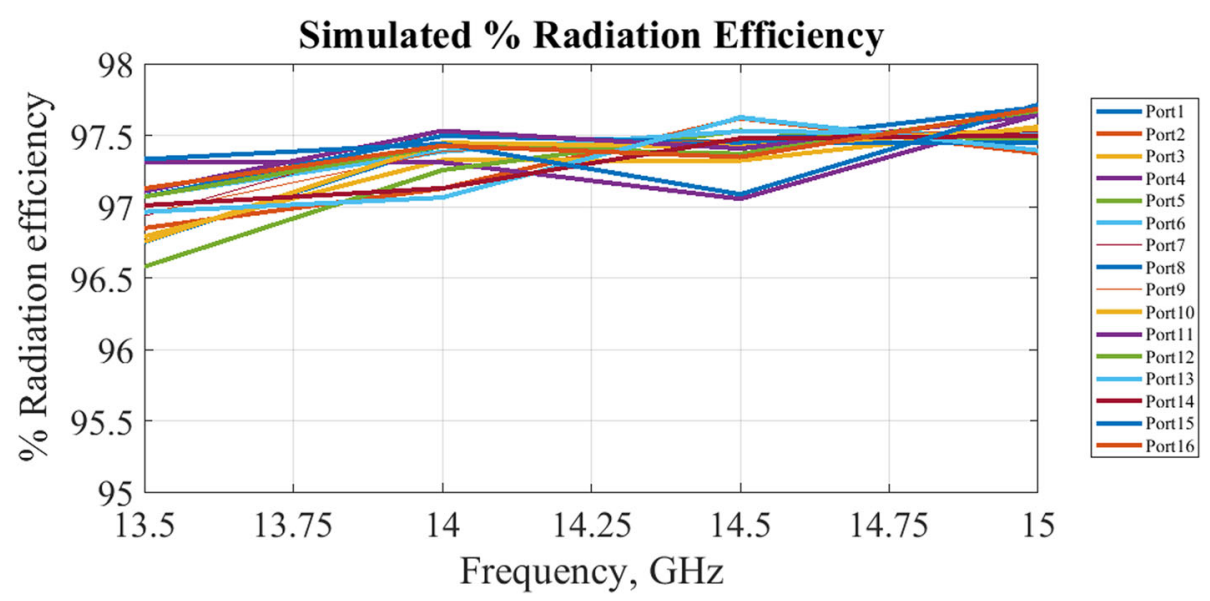

Fig. 6 Simulated percentage radiation efficiency over the 16 ports 


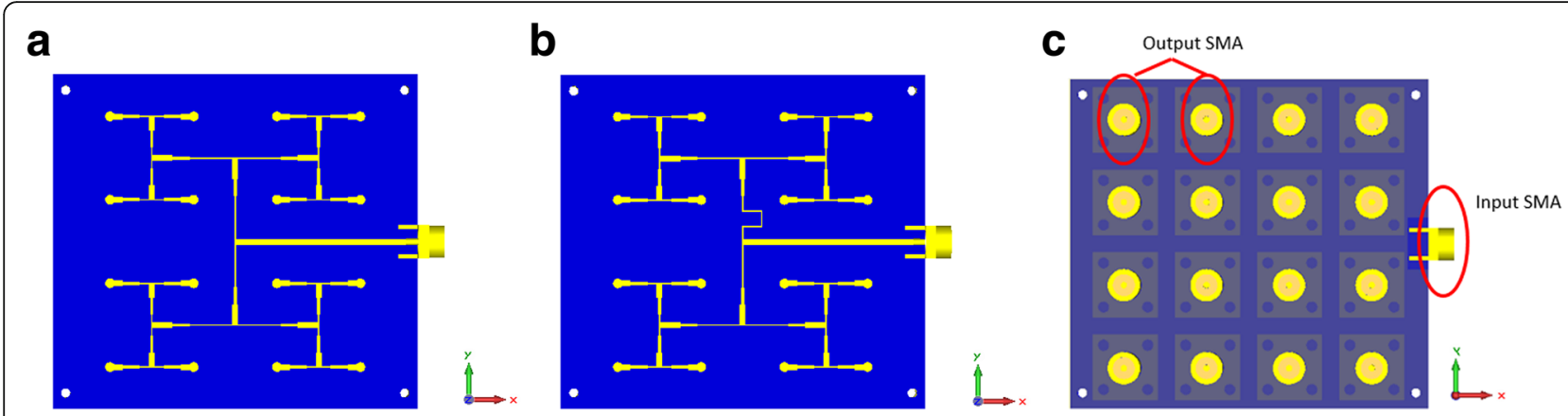

Fig. 7 a Top view of the all $0^{\circ}$ phase network. $\mathbf{b}$ Top view of the $0^{\circ}$ and $180^{\circ}$ phase network. $\mathbf{c}$ Back view of the $0^{\circ}$ and $180^{\circ}$ phase network

passive or active; the key point is that the feeding network needs to produce only two phases to generate multi-directional beams. A switching between several of such beam configurations emulates the effect of beam steering with minimal loss of spatial and angular resolution.

As a starting point, let us consider two equal radiating sources excited in phase opposition and symmetrically located around the $\mathrm{XZ}$ plane at positions of $(0,-\Delta / 2,0)$ and $(0, \Delta / 2,0)$ where $\Delta$ is the interelement spacing. The radiated field produced by these sources will be given by

$$
\begin{aligned}
E(\theta, \phi) & =\vec{E}_{0}(\theta, \phi)\left(e^{j k \sin \theta \sin \phi \frac{\Delta}{2}}-e^{-j k \sin \theta \sin \phi \frac{\Delta}{2}}\right) \\
& =2 j \vec{E}_{0}(\theta, \phi) \sin \left(k \sin \theta \sin \phi \frac{\Delta}{2}\right)
\end{aligned}
$$

if mutual coupling effects are neglected. The sine term will introduce a null on the $\phi=0$ plane and generate a twin lobe pattern with an opposite sign at each side of the null plane. Varying $\Delta$ allows to change the maximum pointing directions to an extent and, if spacing higher than a wavelength is used, multiple lobes on both sides can be obtained. The sine term in (1) is nothing more than the array factor. The element pattern $\vec{E}_{0}(\theta, \phi)$ will limit the resulting directivity for angles with high deviation from boresight. Based on this concept, the proposed fundamental beam-switching scheme that is responsible for the multi-directional beam operation is discussed next.

The building block for the proposed beamswitching scheme rests on physical principle and observation that the direction of the formed beam aligns along the vector connecting the points where $0^{\circ}$ and $180^{\circ}$ phases are fed on a two-element array. An additional degree of freedom is offered by the interelement spacing. By controlling the vector alignment using the two phases, a corresponding directional beam can be attained. This primary building block, based primarily over two antennas, can be systematically mapped and extended to larger arrays to attain multi-beam capabilities. The two-element building block requires that the antennas individually are directional and suitably spaced to avoid grating lobes. Owing to the differential way of excitation, a cancellation null is seen along the boresight and the

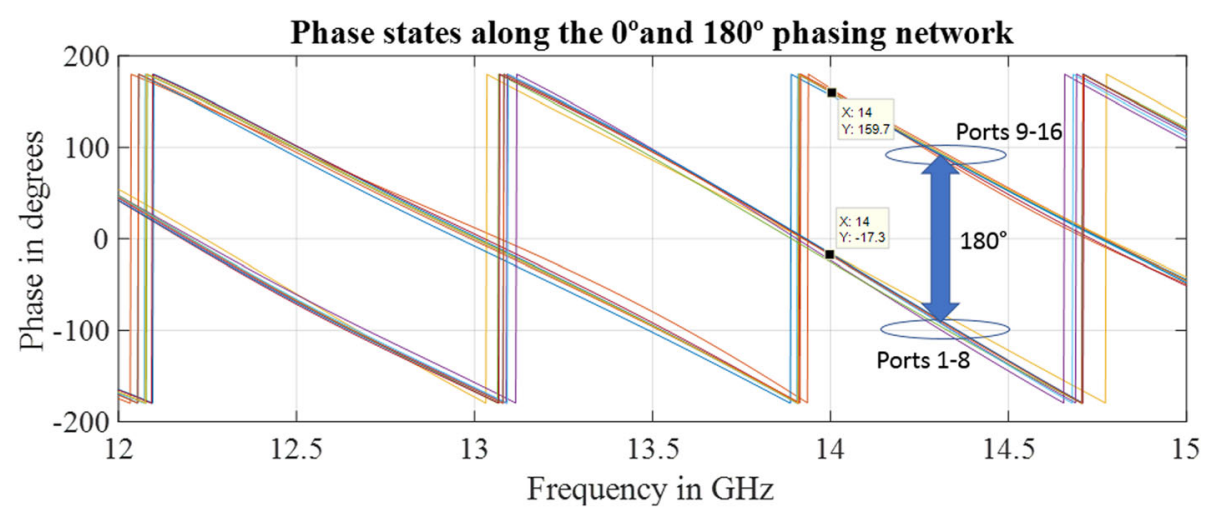

Fig. 8 Phase response of the dual-phasing network of $0^{\circ}$ and $180^{\circ}$ 
Table 1 Demonstration of the two-phase vector alignment beam-switching scheme

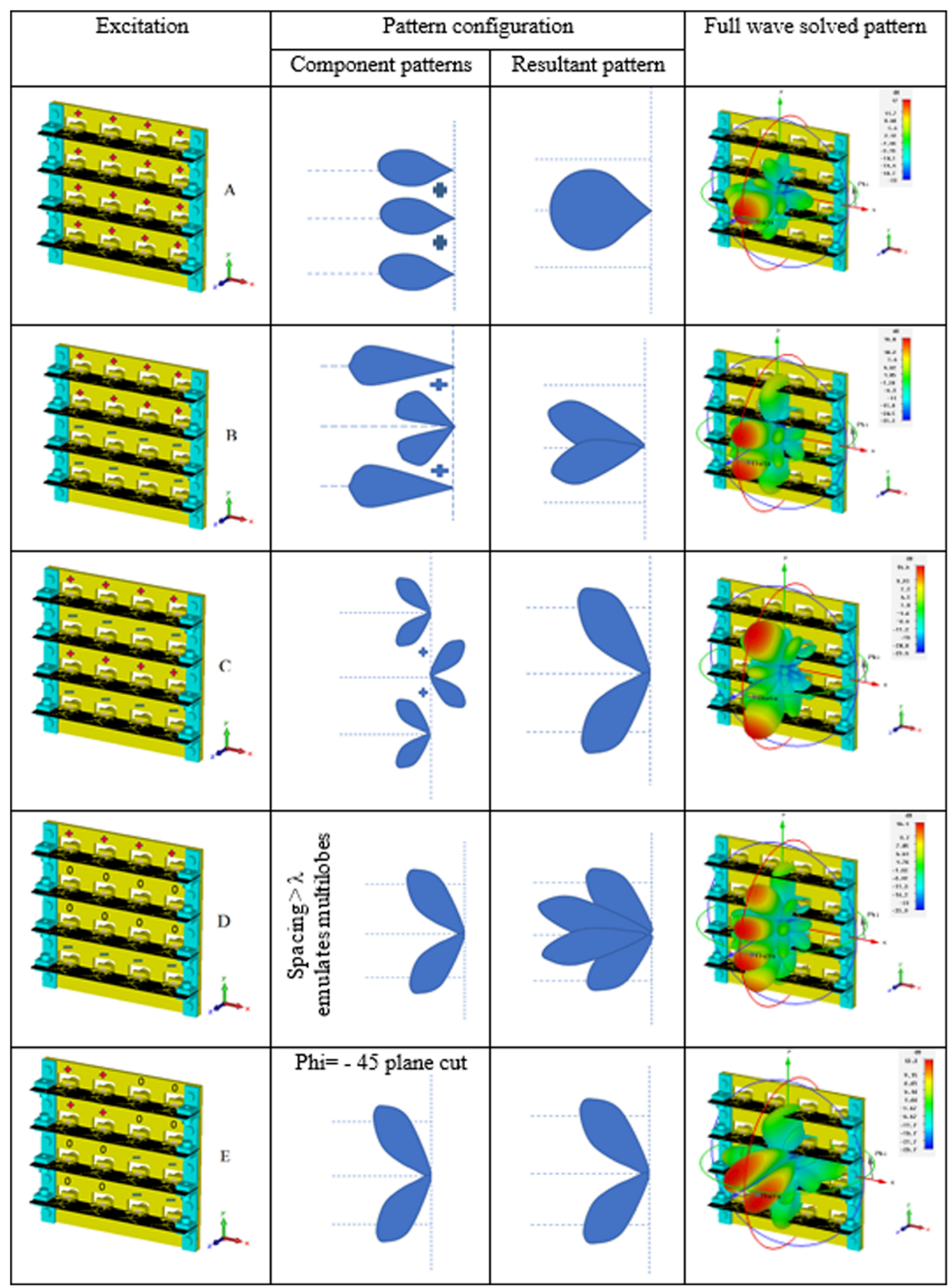

pattern lobes are placed symmetrically around it. The symmetric property arises owing to the oddsymmetrical excitation of the phases. The validation of the building block scheme with a depiction of the corresponding vector alignment and beam directions is shown in Fig. 1 using the basic element presented in [14].

Consider Fig. 1, where two directive antenna elements are placed suitably spaced apart to avoid grating lobes. The individual directive antenna element consists of a printed endfire configuration with additional parasitic elements to enhance the directivity along the endfire direction [14]. A brief description of the antenna element is as follows: The antenna has an operational band from 13.5 to 15 $\mathrm{GHz}$, with a fractional bandwidth of $10.5 \%$. It has one arm of the $\lambda / 2$ dipole printed on one side of the substrate with $\underline{\varepsilon_{r}}=3.55$, loss tangent $(\tan \delta)=0.0027$ and a thickness of $0.508 \mathrm{~mm}$. The other arm is printed on the back side. A printed reflector is added at the point of connection of the SMA to the substrate to improve the front-to-back (F/B) ratio. 
Table 2 Pattern-steering excitation. $K=1 \angle 0,-K=1 \angle 180^{\prime} 0^{\prime}$ implies no port excitation

\begin{tabular}{|c|c|c|c|c|c|}
\hline \multirow{2}{*}{$\begin{array}{l}\text { Port } \\
\text { numbers }\end{array}$} & \multicolumn{5}{|c|}{ Antenna state } \\
\hline & $\bar{A}$ & $B$ & $C$ & $\mathrm{D}$ & $E$ \\
\hline 1 & $k$ & k & k & $k$ & $\bar{k}$ \\
\hline 2 & k & k & k & K & k \\
\hline 3 & k & k & k & k & 0 \\
\hline 4 & k & k & k & K & 0 \\
\hline 5 & k & k & $-k$ & 0 & k \\
\hline 6 & k & k & $-k$ & 0 & k \\
\hline 7 & k & k & $-k$ & 0 & 0 \\
\hline 8 & K & k & $-k$ & 0 & 0 \\
\hline 9 & k & $-k$ & k & 0 & 0 \\
\hline 10 & K & $-k$ & k & 0 & 0 \\
\hline 11 & k & $-k$ & k & 0 & $-k$ \\
\hline 12 & k & $-k$ & k & 0 & $-k$ \\
\hline 13 & k & $-k$ & $-k$ & $-k$ & 0 \\
\hline 14 & k & $-k$ & $-k$ & $-k$ & 0 \\
\hline 15 & k & $-k$ & $-k$ & $-k$ & $-k$ \\
\hline 16 & K & $-k$ & $-k$ & $-k$ & $-k$ \\
\hline
\end{tabular}

The radiation from the dipole being omnidirectional is made directional by the reflector and by the parasitic elements that couple the dipole electric field along the substrate plane. It has a return loss of $\geq$ $10 \mathrm{~dB}$ in the band $13.5-15 \mathrm{GHz}$. The endfire antenna element is inspired by the energy-focusing property of graded-index optical fibres.

Each antenna element is fed by a phase of $0^{\circ}$ and $180^{\circ}$, respectively, with the same unity amplitude. Such an excitation results in the formation of a two- directional pattern along the cut normal to the two dielectric planes (Phi90). The 3D antenna pattern and its $2 \mathrm{D}$ projection are shown in Fig. 1. A cancellation beam resulting in the formation of a null divides the pattern into two symmetrical halves. The orientation of the pattern is aligned along the vector connecting the two-phase feeding points. This gives scope to develop a multi-beam antenna array that utilizes a visual representation of the vectors and their correspondingly mapped 3D patterns. The orientation of the beams can be symmetrically controlled for varied interelement distances. A study of the interelement spacings and the dependence of the formation of nulls and the pattern beams on it is shown in Fig. 2 which represents the $\phi=90^{\circ}$ pattern cut for the two-element antenna in Fig. 1 for varied spacings of $0.25-1.5 \lambda$. As can be seen in Fig. 2, the asymmetrical phase excitation results in a wellpronounced deep null along the boresight independent of the spacing. This shows that the antenna using this methodology is a suitable candidate for applications, mainly in NLOS and in applications requiring oblique beams with no beams along boresight. A direct observation from the parametric variation in Fig. 2 is that the pointing angles of the first beams located symmetrically across the boresight nulls move away from the boresight as the interelement spacing decreases. Also, the beamwidth increases with decrease in interelement spacing. For spacings, larger than $\lambda$, multi-beams appear which, when used in larger arrays, can lead to constructive use as multiple lobes of similar magnitude. As a fullwave electromagnetic solver is used, the mutual coupling effects are taken into account in Figs. 1 and 2 .
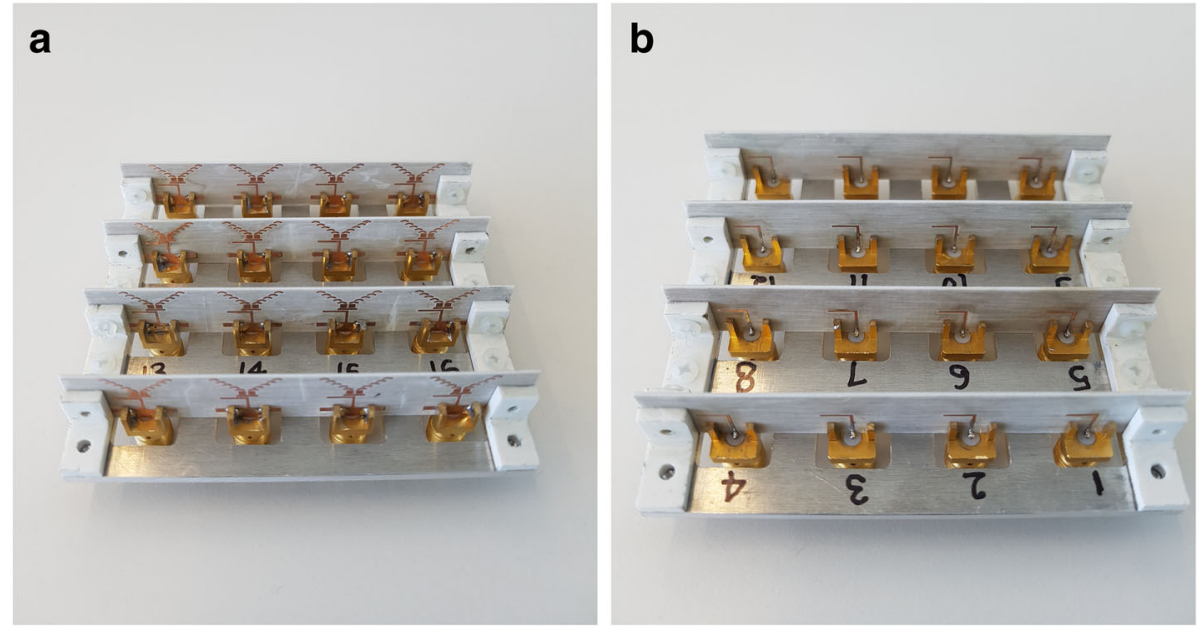

Fig. 9 Fabricated $4 \times 4$ array. a Front view. b Back view 


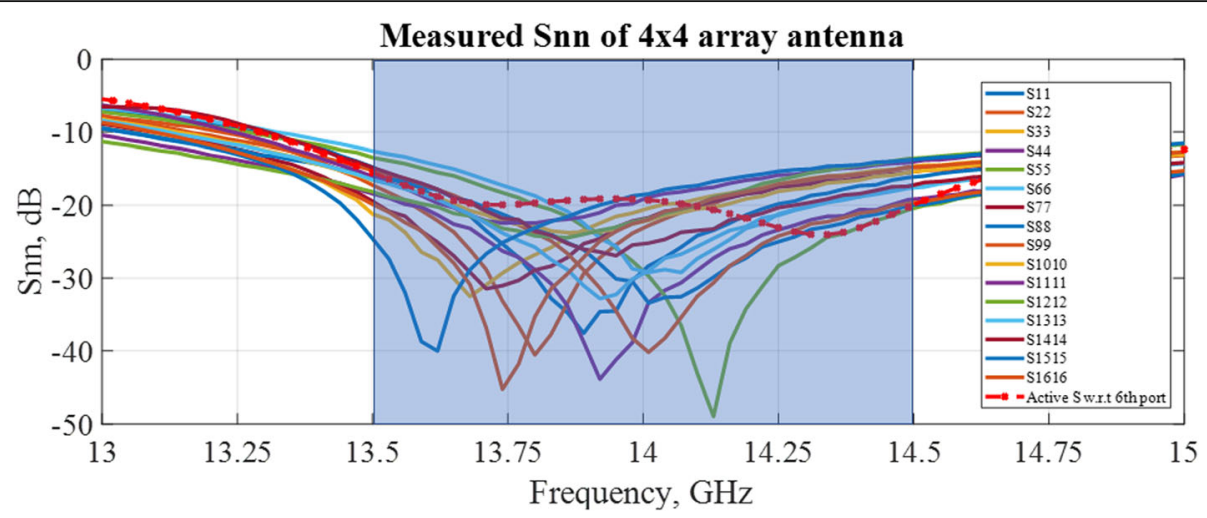

Fig. 10 Measured $S$ parameters compared with active $S$ parameter with respect to port 6

\subsection{Antenna array design}

The antenna element described in [14] was used in the $4 \times 4$ sub-array design as shown in Fig. 3 . A $4 \times$ 4 subarray validation is chosen as a precursor for an $8 \times 8$ array operation. As the proposed beamswitching scheme has an additive symmetry, the $4 \times$ 4 subarray deductions can be extended onto the $8 \times$ 8 without loss of generality. The antenna array interelement spacing was set to $0.8 \lambda$ lying within the $0.5 \lambda$ to $\lambda$ regime to avoid the onset of grating lobes for boresight pattern.

The antenna array was simulated using full-wave simulations for $S$ parameters and the individual antenna patterns computed were subjected to pattern post-processing to test the beam-switching scheme. The port to port isolation throughout the array was better than $25 \mathrm{~dB}$. The simulated reflection coefficients at the 16 ports are shown in Fig. 4 and the port-to-port isolation with respect to a selected port (port1) are shown in Fig. 5.

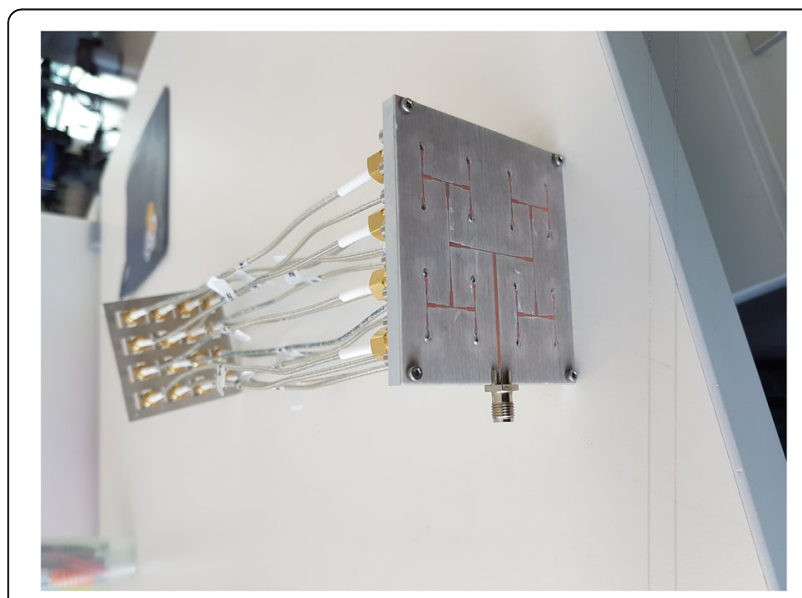

Fig. 11 Fabricated equal phase network
From Fig. 4, a similar return loss characteristic for the 16 antennas is seen which is essential in realizing the near identical pattern multiplication with the $4 \times 4$ array factor to obtain the switched beams. Figure 5 represents a good port-to-port isolation $(<-$ $25 \mathrm{~dB}$ ) which helps in isolating the individual patterns and reduces mutual coupling that may influence the current distribution on the neighbouring antenna elements. The simulated radiation efficiency along the embedded ports is shown in Fig. 6 . Around the band $13.5-15 \mathrm{GHz}$, the simulated percentage of radiation efficiency varies between 96.5 and 98 .

\subsection{Phase-shifting feed network design}

Due to the nature of the proposed beam-switching scheme in Section 2.1 and in order to validate experimentally the approach, a two-state phase feeding network is necessary to feed into the elements of the antenna array. Since the proposed scheme is a phase only, the amplitudes are set to be uniformly distributed over the array. This equal amplitude distribution can be realized by a corporate feeding network extended to a $4 \times 4$ array as shown in Fig. 7. The phase-shifting network in Fig. 7a consists of tailored transmission lines printed on a $\underline{\varepsilon_{r}}=3.55$ substrate of a thickness of $0.508 \mathrm{~mm}$. The methodology adapted for the realization of the feeding network involved identifying the positions of the 16 antenna elements in the antenna array and working the feedline backwards to the source. The source port impedance of $50 \Omega$ is transitioned over $100-\Omega$ branching lines along with the use of quarter wave transformer at $14 \mathrm{GHz}$ to realize the network. The $4 \times 4$ array was symmetrically divided into four sections of $2 \times 2$ each and the designed feeding line for a $2 \times 2$ network was mirrored symmetrically across the other three sections so that the phase component retains its 

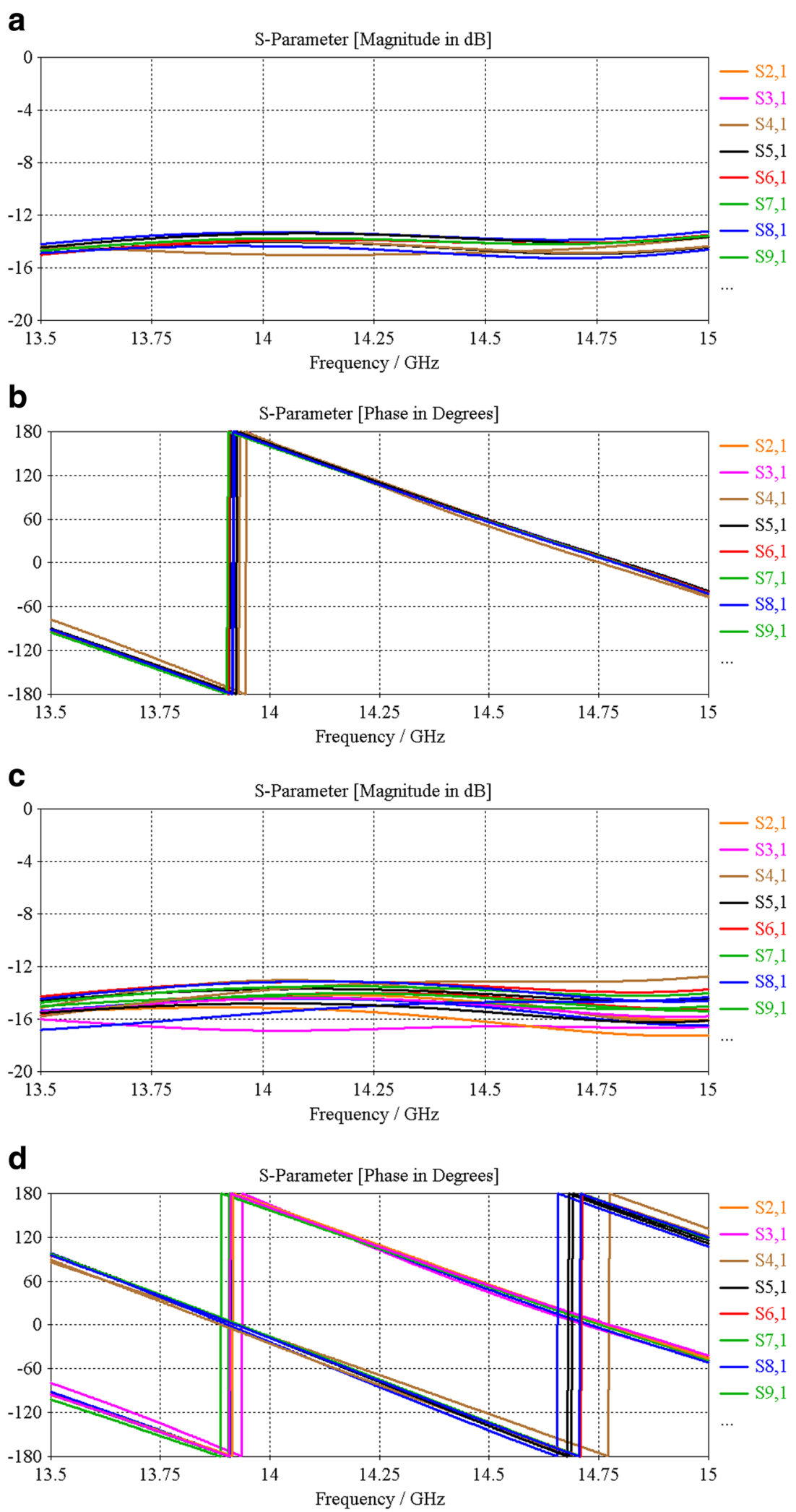

Fig. 12 a Frequency response of the magnitude of Sij of the $0^{\circ}$ phasing network (simulated). $\mathbf{b}$ Frequency response of the phase of Sij of the $0^{\circ}$ phasing network (simulated). c Frequency response of the magnitude of Sij of the $0^{\circ} / 180^{\circ}$ phasing network (simulated). $\mathbf{d}$ Frequency response of the phase of Sij of the $0^{\circ} / 180^{\circ}$ phasing network (simulated) 


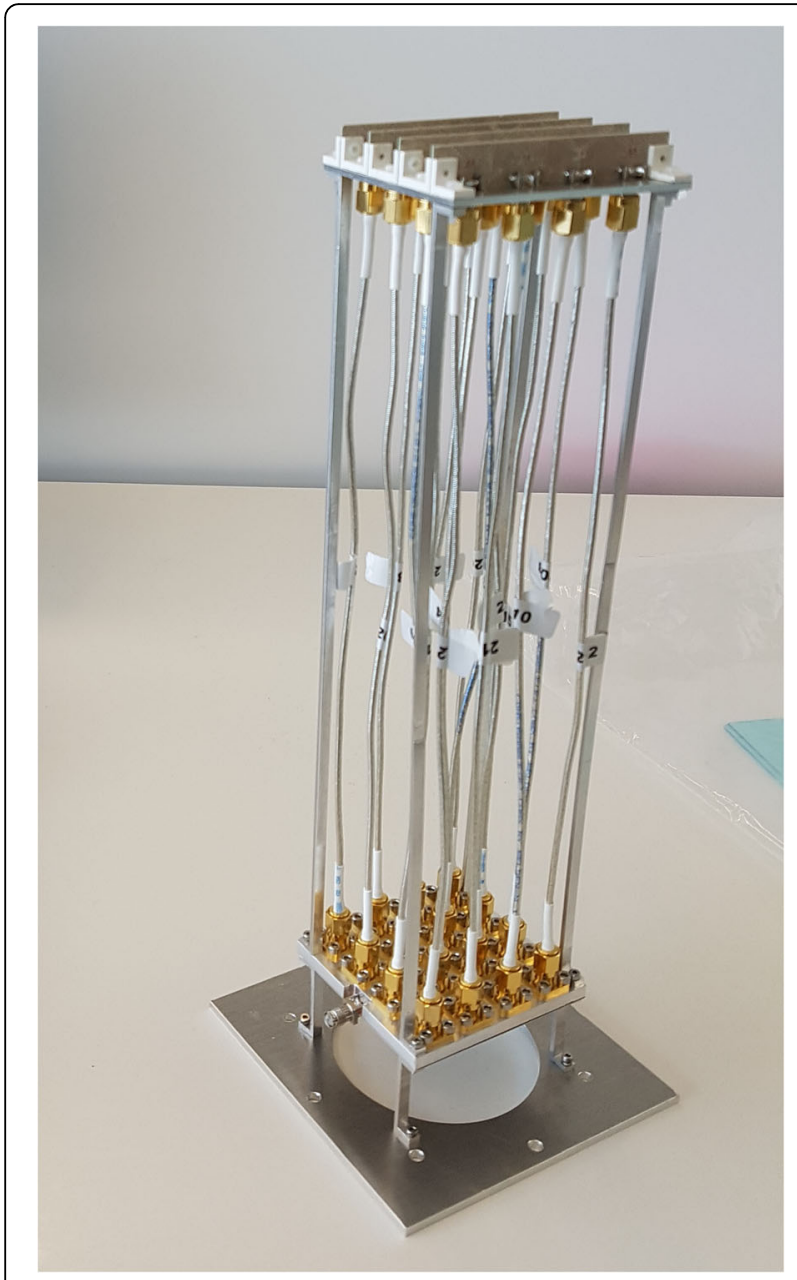

Fig. 13 Fabricated antenna array with phasing network

consistency along the line. A similar approach was followed in realizing the phase network of Fig. 7b but introducing an additional phase length of $180^{\circ}$ using an $U$-bend amounting to the additional $180^{\circ}$ phase shift across half of the network. The designed network therefore had 8 output ports at $0^{\circ}$ and other 8 output ports at $180^{\circ}$ as in the phase response shown in Fig. 8. As the designed beam-switching scheme requires only two-phase states, such a phase feeding network was deemed both simple and costeffective from the experimental validation point of view. Figure $7 \mathrm{c}$ depicts the back view of the phase feeding network which consists of SMA connectors feeding onto the ports of the phasing network. An edge-fed SMA connector was used as the primary source to feed into the phasing network.

The two-element array building block can be used to construct the multi-beam operation by considering the constructive and destructive sequential addition of the two-element pattern. Analytical

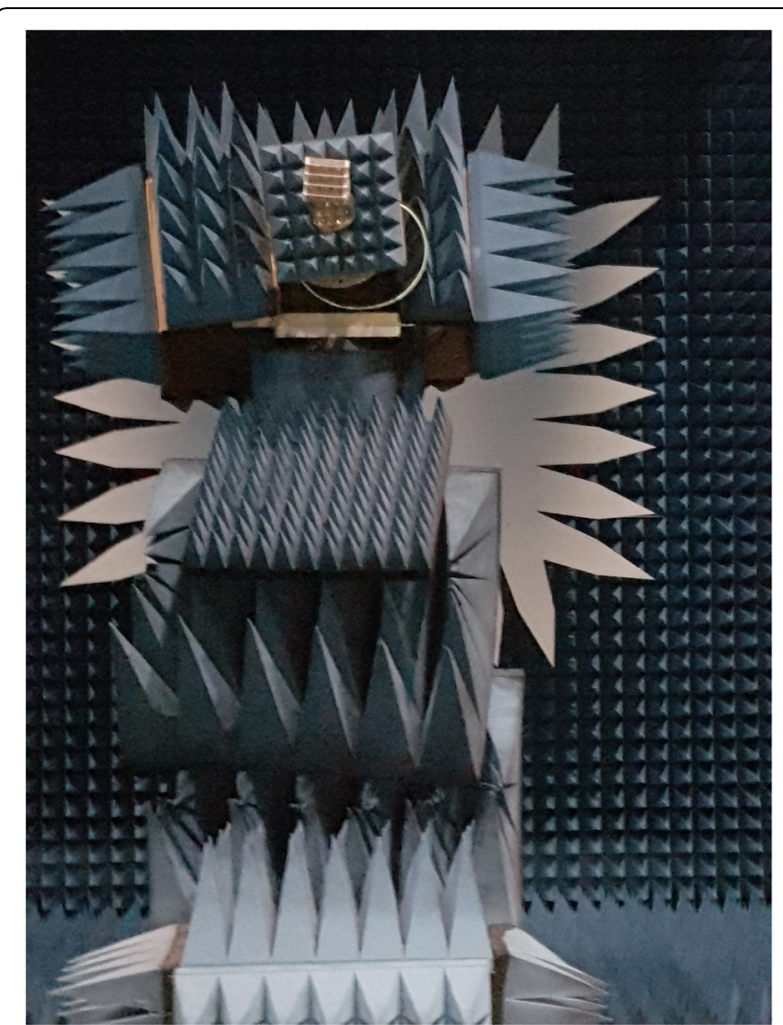

Fig. 14 Pattern measurement of the $4 \times 4$ antenna array in anechoic chamber

descriptions quantifying the visual intuitive approach depicted in Table 1 for each of the five cases are shown in Eqs. 2, 3, 4, 5, 6, 7 and 8. Table 1 explains the way of deriving patterns $\mathrm{A}-\mathrm{E}$ by their component patterns.

Coming back to the simple model based on the array factor, the general field pattern to derive the cases $\mathrm{A}-\mathrm{C}$ and $\mathrm{E}$ is given by Eq. 2, as follows:

$$
\begin{aligned}
E(\theta, \phi)=\vec{E}_{0}(\theta, \phi) & \left(\sum_{i=0}^{1} a_{i} e^{j k \sin \theta \sin \phi(2 i-1) d y}\right. \\
& \left(\sum_{l=0}^{1} b_{l} e^{j k \sin \theta \sin \phi\left(l-\frac{1}{2}\right) d y}\right. \\
& \left.\left.\left(\sum_{m=0}^{3} c_{m l} e^{j k \sin \theta \cos \phi\left(m-\frac{3}{2}\right) d x}\right)\right)\right)
\end{aligned}
$$

where the summation term with $c_{m l}$ represents the contribution of a centred linear array of four elements spaced $d x$ along $x$, the summation term with $b_{l}$ coefficients accounts for the contribution of two centered linear arrays of the previous type spaced $d y$ along $y$ and finally the summation term with $a_{i}$ coefficients gives rise to two sets of $2 d y$ spaced 


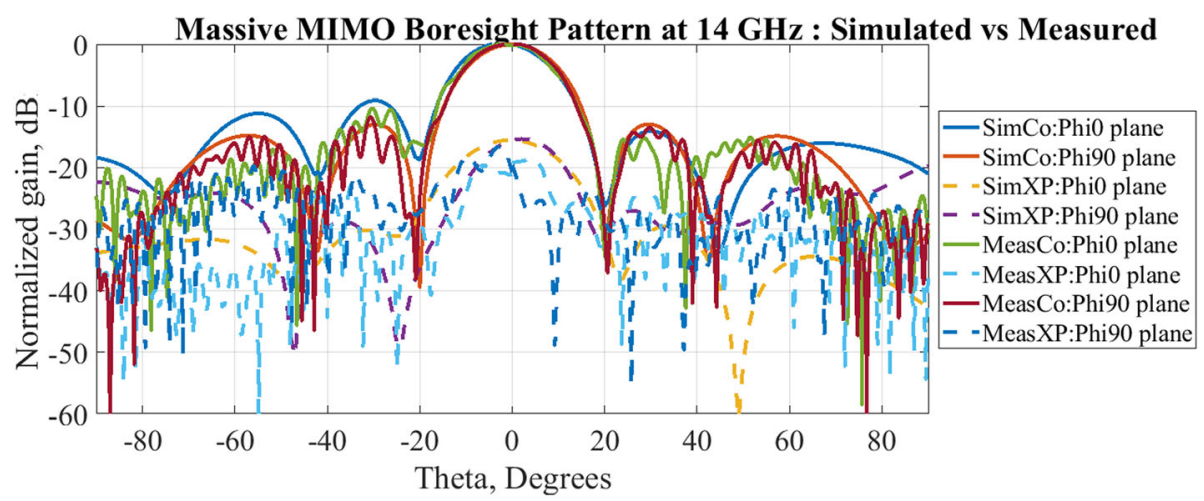

Fig. 15 Simulated vs measured pattern state A

along $y$ linear array of the previous type row pairs. The above-nested equation opens up in layers over the following progression of field calculations: starts from a linear array of four elements with the origin at its middle point, uses it to build two linear arrays centred around the origin, and finally, by an appropriate symmetrical displacement of these last two towards the edges of the $4 \times 4$ array geometry, the field, neglecting coupling between elements, is obtained. This entire process when suitably excited as in the two-state only weighting scheme below results in the realization of multiple beams that can be cast towards pre-defined directions. The coefficients for cases $\mathrm{A}-\mathrm{C}$ and $\mathrm{E}$ in Table 1 are given by

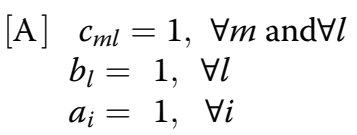

[B] $\quad c_{m l}=1, \forall m$ and $\forall l$

$b_{l}=1, \quad \forall l$

$a_{i}=(-1)^{i+1}, \quad \forall i$
$[C] \quad c_{m l}=1, \forall m$ and $\forall l$

$b_{l}=(-1)^{l+1}, \forall l$

$a_{i}=1, \quad \forall i$

$\begin{aligned} \text { [E] } c_{m l} & =\left\{\begin{array}{l}1 \quad\left\{\begin{array}{l}m=0,1 \text { and } l=1 \\ m=2,3 \text { and } l=0\end{array}\right. \\ 0 \quad \text { otherwise }\end{array}\right. \\ b_{l}=1, \forall l & \forall i=(-1)^{i+1}, \quad \forall i\end{aligned}$

The special case $\mathrm{D}$ is defined by Eq, 7:

$$
\begin{aligned}
E(\theta, \phi)=\vec{E}_{0}(\theta, \phi) & \sum_{l=0}^{1} b_{l} e^{j\left(k \sin \theta \sin \phi(2 l-1) \frac{3 d d y}{2}\right.} \\
& \sum_{m=0}^{3} c_{m l} e^{j\left(k \sin \theta \cos \phi\left(m-\frac{3}{2}\right)\right) d x}
\end{aligned}
$$

with coefficients given by

$$
\begin{aligned}
& \text { [D] } \quad c_{m l}=1, \forall m \text { and } \forall l \\
& b_{l}=(-1)^{l-1}, \quad \forall l
\end{aligned}
$$

(4) Pattern A is the classical boresight beam which uses a uniform amplitude and phase excitation to obtain the

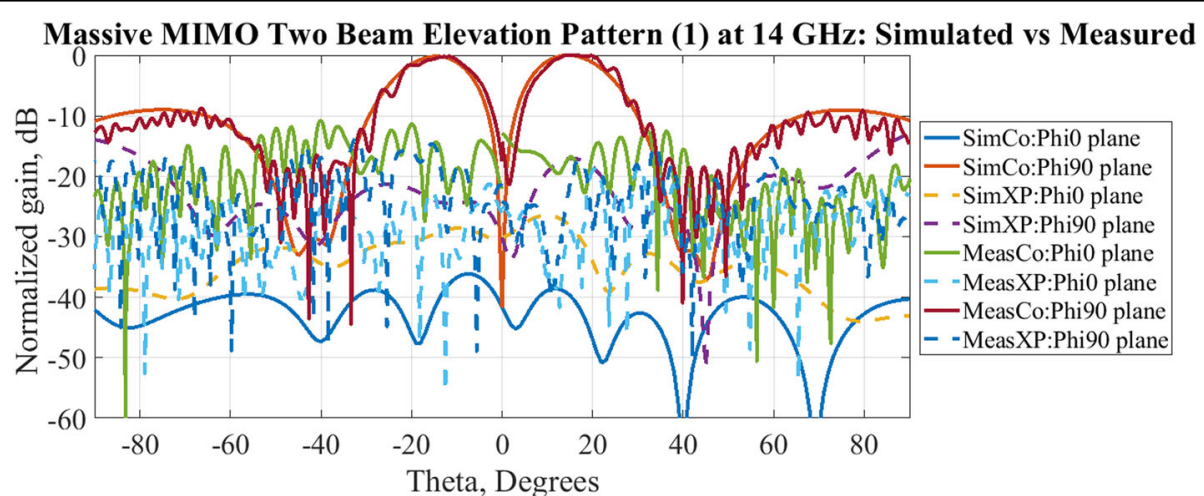

Fig. 16 Simulated vs measured pattern state B 


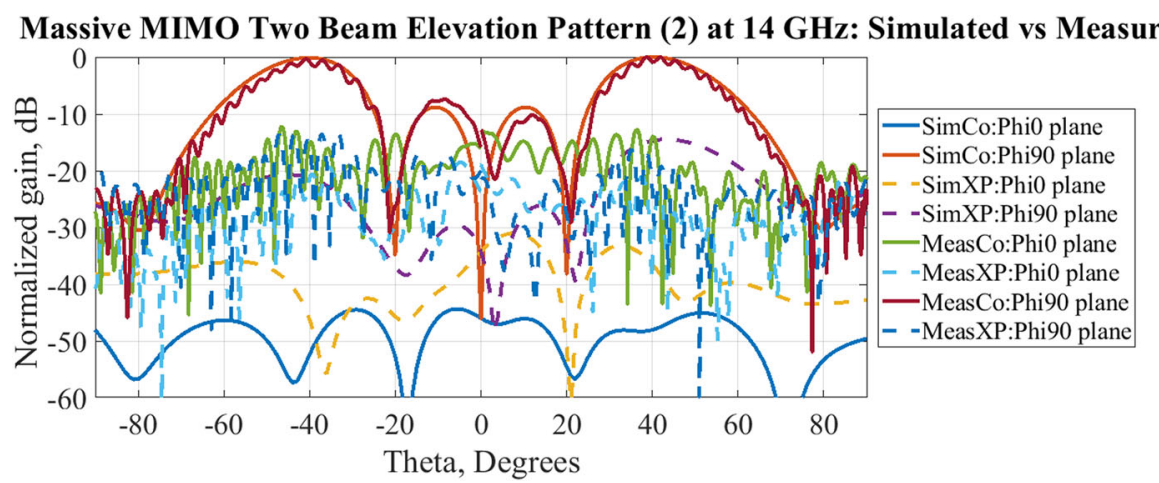

Fig. 17 Simulated vs measured pattern state C

directional beam along the boresight. The mechanism of beam formation is depicted in the second column as an addition of 3 individual beams formed respectively from array rows 1 and 2, array rows 2 and 3 and array rows 3 and 4 . Each of these rows has uniform amplitude excitation and equal phase of zero. Row 1 and row 2 add up constructively so does 2 and 3, and 3 and 4. The resultant beam is a main beam in the boresight with an increased amplitude.

Pattern B depicts an excitation where row 1 and row 2 are fed with a phase $0^{\circ}$ and row 3 and row 4 are fed with a phase of $180^{\circ}$. Going by the previous analogy of splitting the 4 rows into 3 groups of 2 . Row 1 and row 2 excitation produces a single main lobe. As in the discussion in Section 2.1, row 2 and row 3 being fed by opposite vectors produces a split beam with reduced amplitude. Row 3 and row 4 excitation produces the same main lobe as row 1 and row 2. The resultant of the three beams can be viewed as the split central beam whose magnitude is being enhanced by the end beams due to rows 1 and 2 and rows 3 and 4 as depicted in column 3 .
Pattern $\mathrm{C}$ depicts an alternating opposite vector excitation along rows $1,2,3$ and 4 . Thus, rows 1 and 2 and 3 and 4 give rise to a split beam. Rows 2 and 3 also give rise to a split beam but the vector alignment is in the opposite direction thus effectively acting as a cancellation to the boresight pointing beam of the split beams of rows 1 and 2 and 3 and 4 . The resultant is a beam cancelled over wider angle along the boresight and symmetrically obliquely oriented as in column C4.

Pattern D is used to illustrate that owing to the selection of phases and combined use of thinning of the array, an extra dimension of control, exploiting the interelement spacing can be used to attain multiple lobes when an opposite vector excitation is used. In the pattern, row 1 is a phase tethered with row 4 and with row 2 and row 3 match terminated. This emulates a thinned planar array scenario and leads to a four-beam multiple patterns. As a theoretical rule, the number of multiple lobes formed increases with the number of thinned rows.

Pattern E is used to demonstrate that the proposed concept can be used to orient twin symmetric beams

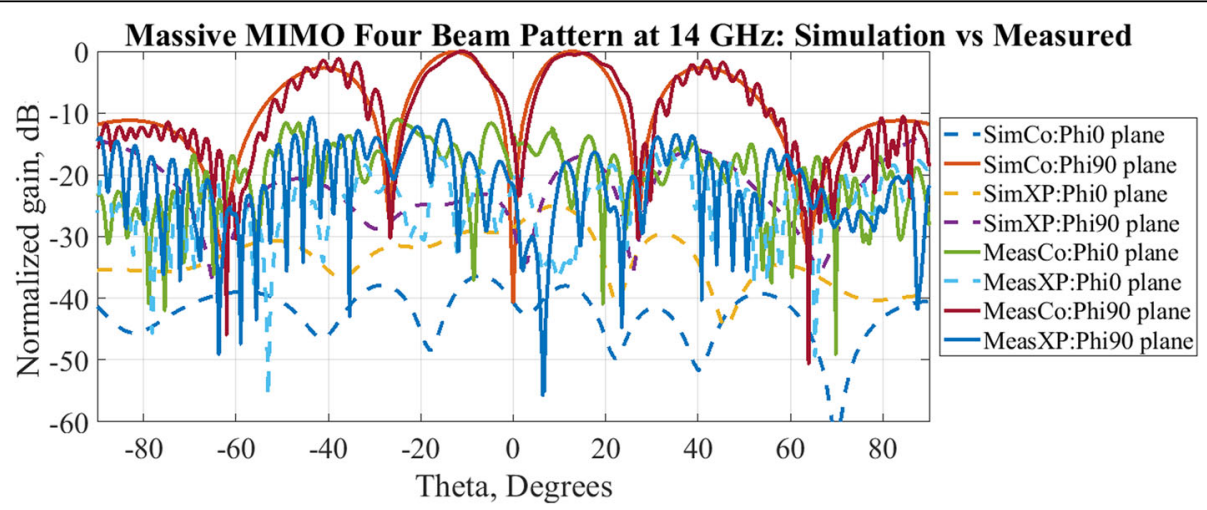

Fig. 18 Simulated vs measured pattern state D 


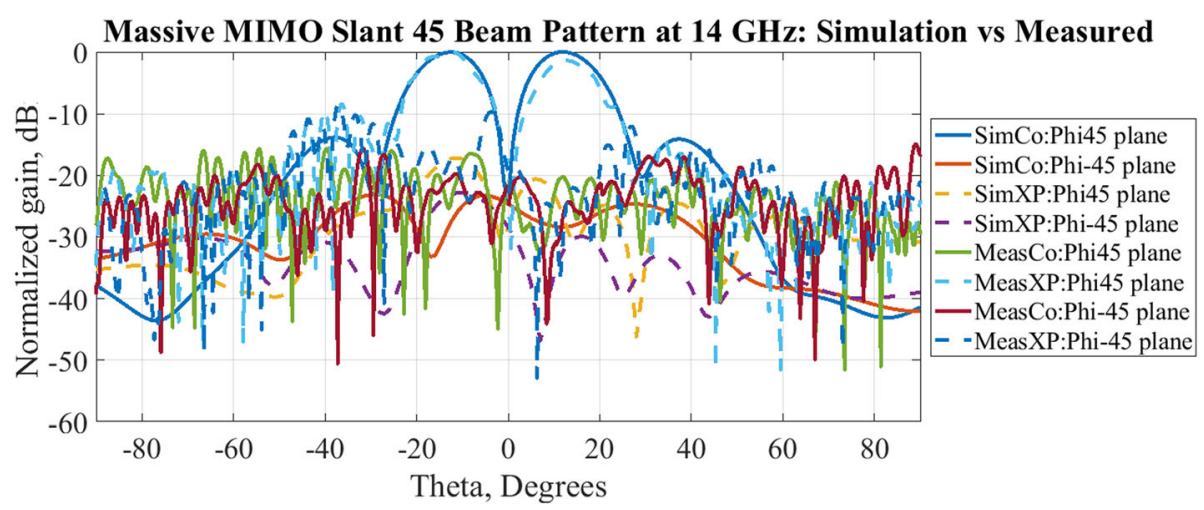

Fig. 19 Simulated vs measured pattern state $E$

along other desired direction using the vector alignment rule discussed in Section 2.1. In excitation E, the vector alignment is along the phi $=-45$ plane through a selected $2 \times 2$ sub-array feeding, combined with $2 \times 2$ sub-array and a thinning over the other $2 \times 2$ sub-arrays along the other diagonal. This leads to a beam oriented along the $-45^{\circ}$ direction as in column 4 of pattern $\mathrm{E}$.

With the above five states that have been demonstrated and experimentally validated in the following discussion, other multi-beam states using the proposed scheme can be realized by one or more combinations of pattern $\mathrm{A}-\mathrm{E}$ which in turn are obtained from the fundamental two-element split beam block.

\subsection{Excitation matrices of the beam-switching scheme}

The different matrices with the antenna excitations as individual elements can also be expressed in the matrix form as shown below. These matrices are related to each other by simple matrix operations.

$$
\begin{aligned}
A & =\left[\begin{array}{llll}
k & k & k & k \\
k & k & k & k \\
k & k & k & k \\
k & k & k & k
\end{array}\right] \\
B & =\left[\begin{array}{cccc}
k & k & k & k \\
k & k & k & k \\
-k & -k & -k & -k \\
-k & -k & -k & -k
\end{array}\right] C=\left[\begin{array}{cccc}
k & k & k & k \\
-k & -k & -k & -k \\
k & k & k & k \\
-k & -k & -k & -k
\end{array}\right] \\
D & =\left[\begin{array}{cccc}
k & k & k & k \\
0 & 0 & 0 & 0 \\
0 & 0 & 0 & 0 \\
-k & -k & -k & -k
\end{array}\right] E=\left[\begin{array}{cccc}
k & k & 0 & 0 \\
k & k & 0 & 0 \\
0 & 0 & -k & -k \\
0 & 0 & -k & -k
\end{array}\right]
\end{aligned}
$$

Table 2 depicts the antenna state and gives a variation of the excitation vector over the 16 ports. It can be clearly seen that it is a two-vector variation.

\section{Antenna array measurements and discussion}

The antenna array and phasing network that was discussed in Section 2.2 and 2.3 was fabricated. The fabrication process involved the preparation of the CAD files and the PCB printing was performed through LPKF protomat on a dielectric sheet of thickness $0.508 \mathrm{~mm}$ and

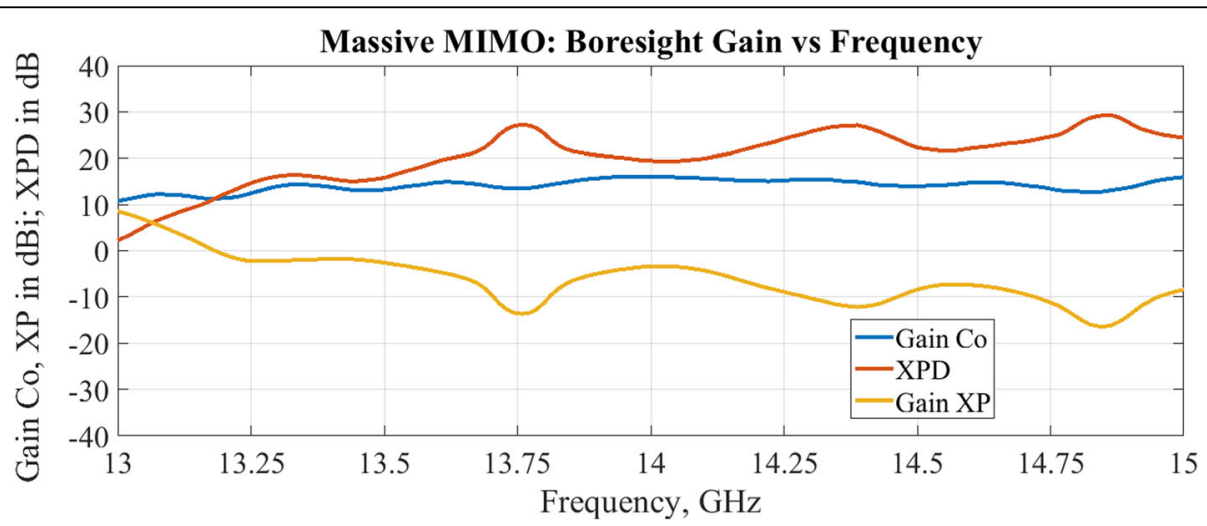

Fig. 20 Measured gain vs frequency pattern state A 


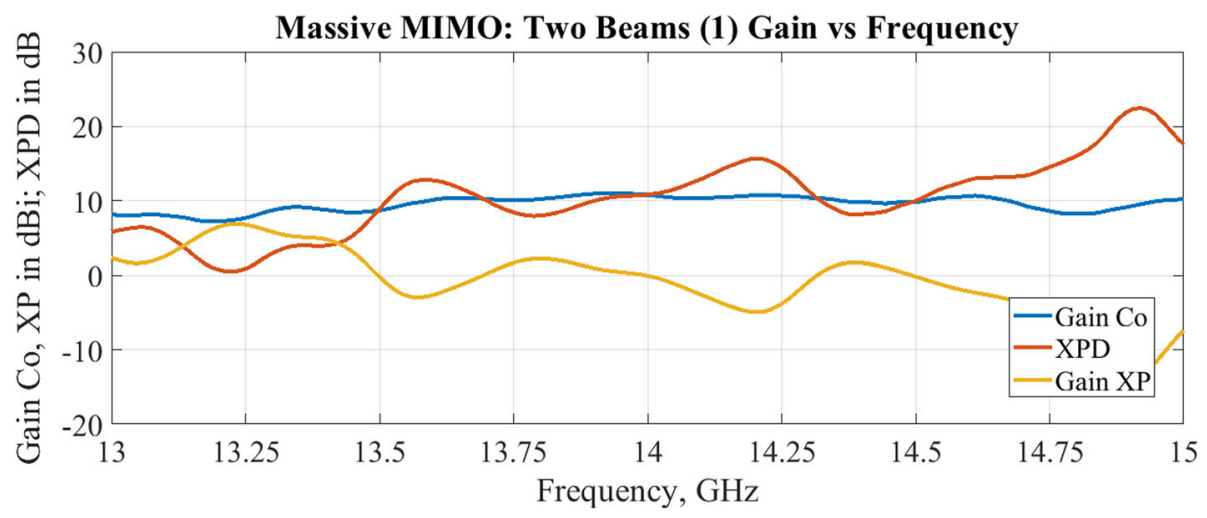

Fig. 21 Measured gain vs frequency pattern state B

$\varepsilon_{r}=3.55$. The back plate of the individual element made of aluminium was extended to span the area of $4 \times 4$ array footprint. Each of the group of four antenna elements that lie along the XZ plane was printed onto a common dielectric substrate with L holder PVC supports at the edges. The common dielectric support for a group of four equally spaced elements eliminates the need to introduce $\mathrm{L}$ holders periodically five times over the $\mathrm{YZ}$ plane thereby minimizing dielectric (PVC) influence. The SMA connectors are edge-fed. They are supported by the $\mathrm{L}$ holders and the dielectric, that rest on the aluminium back plate encompassing the $4 \times 4$ array footprint as shown in Fig. 9. Figure 9a shows the front view and Fig. 9b shows the back view of the fabricated $4 \times 4$ antenna array.

The antenna array in Fig. 9 was measured for its $S$ parameters and this is depicted in Fig. 10. The active reflection coefficient with respect to port 6 for the boresight case derived from individual ports Snn parameters is compared with them in Fig. 10. For boresight beam conditions, from [15], the active reflection coefficient at a port is the summation of the individual Snn values added onto the port of interest. For other angles of incidence, the active reflection coefficient changes.

In Fig. 10, the case considered is for a direct boresight incidence. It can be seen that the return loss at each of the ports is better than/well over $10 \mathrm{~dB}$ over the band of $13.5-14.5 \mathrm{GHz}$.

The phase feeding network discussed in Section 2.3 was fabricated and characterized individually before feeding on to the antenna array. The fabricated feed network for all the $0^{\circ}$ phases is shown in Fig. 11. The simulated frequency response for the phase feeding network for input and output ports is shown in Fig. 12. The measured phase response was close to the simulated one in Fig. 8 and a balanced amplitude and phase operation were seen.

The antenna array and the phase feeding network were aligned and assembled with the help of a supporting structure, and semi-rigid cables (whose losses were characterized in the post-processing) were used to connect the feed network to the antenna array. A manual replacement and

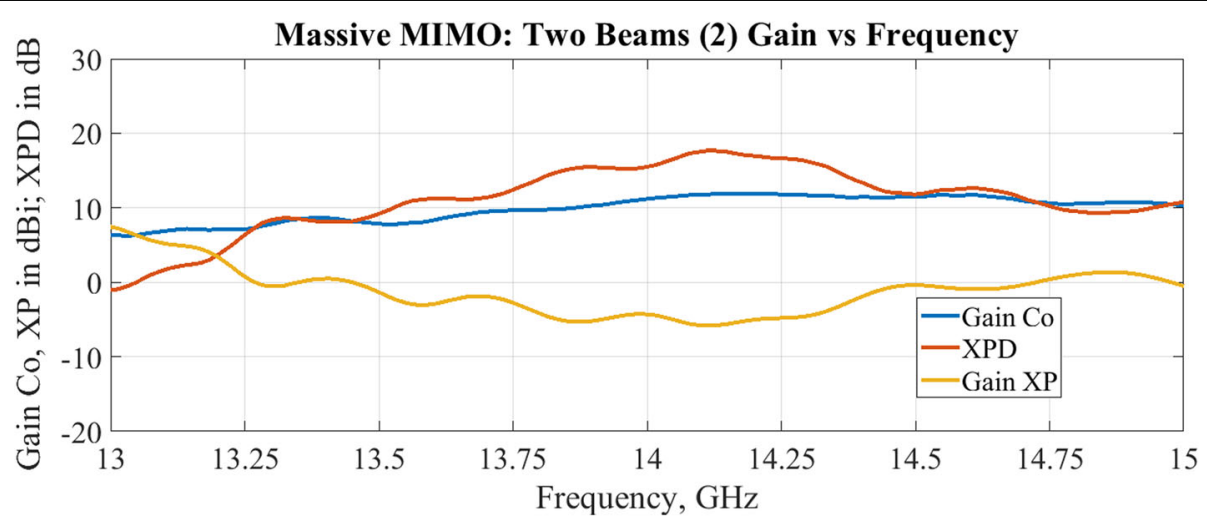

Fig. 22 Measured gain vs frequency pattern state $C$ 


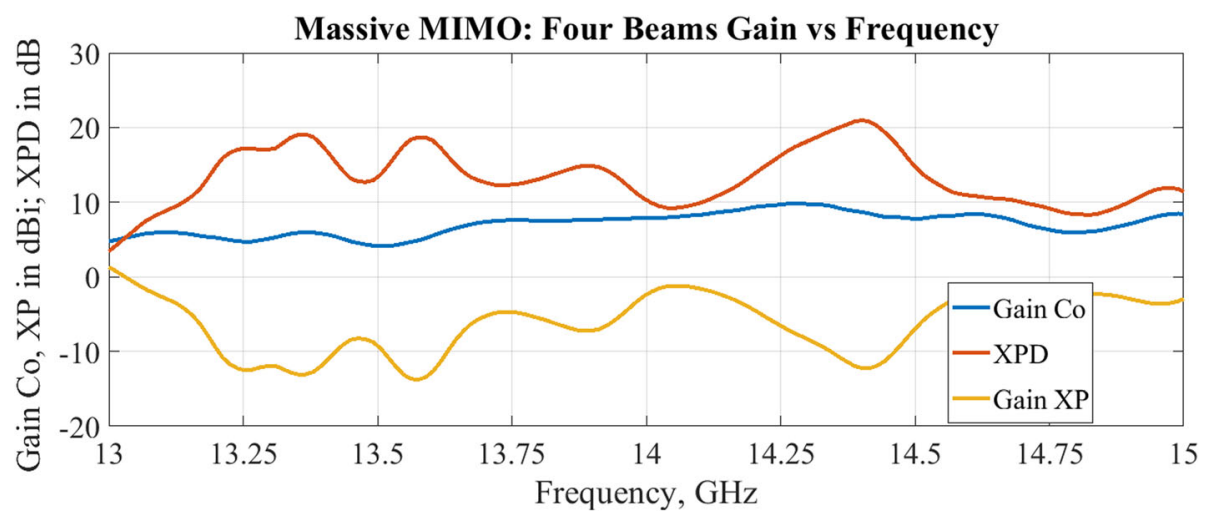

Fig. 23 Measured gain vs frequency pattern state $D$

reconfiguring of the cable was performed to switch between the patterns. All open ports realized from such a reconfiguration were terminated with matched $50-\Omega$ loads to emulate real working conditions. The complete assembled antenna is shown in Fig. 13.

Figure 14 depicts the antenna setup for pattern measurement in the anechoic chamber. Since most of the beams to be measured were designed to have a cancellation beam at boresight, the beam pointing angle of boresight case was used as reference in calibration with an alignment of the roll axis for the measurement of other patterns. The measured antenna pattern is compared with the simulation for cases $\mathrm{A}-\mathrm{E}$ and is presented in Figs. 15, 16, 17, 18 and 19. A good cross-polar discrimination (XPD) is obtained for beamwidths around $40^{\circ}$ for cases A-E. The dependency of the co-polar gain, cross-polar gain (XP) and XPD on frequency for the different cases was also measured for the different antenna states of A-E using the two-antenna method. These measurements are depicted in Figs. 20, 21, 22, 23 and 24. It is important to note that the antenna beam patterns were stable throughout the operating bandwidth of the antenna for the phase excitations.

\section{Conclusion}

A method to realize multiple beam operation using twophase configuration was demonstrated. The beamswitching scheme facilitates the control of the geometric planes onto which the beams are projected. A complete characterization of the fabricated $4 \times 4$ antenna subarray in terms of its active $S$ parameters and antenna pattern was presented. The antenna array has a gain of $16 \mathrm{dBi}$ for a $4 \times 4$ configuration in case A (boresight). The proposed beam-switching scheme and the antenna array can find potential application in scenarios where enhanced multi-user perception is required such as in densely crowded stadiums or in custom-designed 5G small cell scenarios. Another capability of this beamswitching scheme is to cast beams along NLOS which can be of potential use given the recent interest in NLOS communications in millimetre-wave massive MIMO. Finally, the method proposed introduced a building block and a methodology for using it to construct directional multiple beams. The antenna array

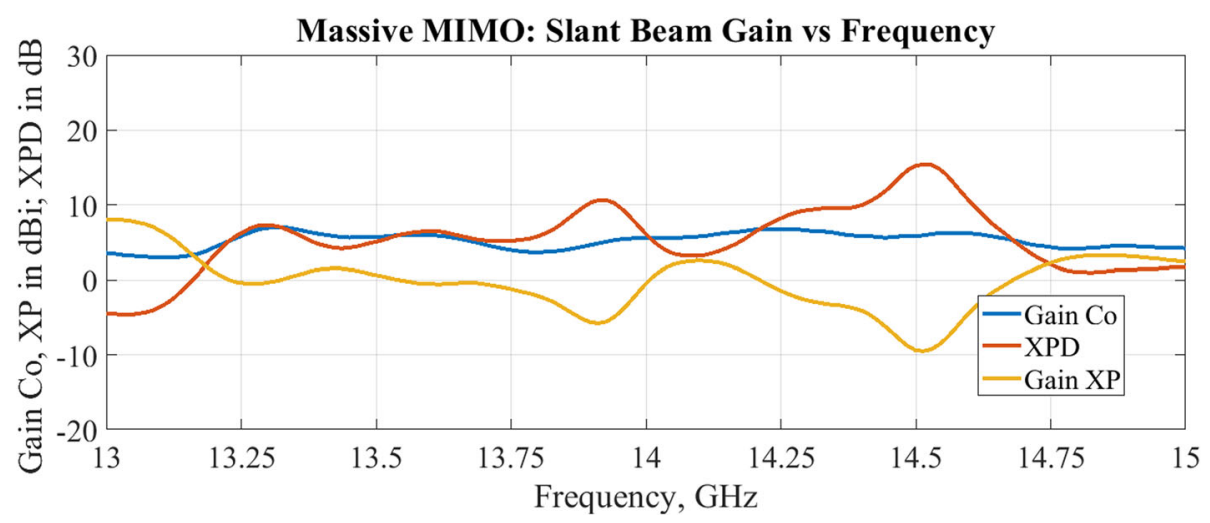

Fig. 24 Measured gain vs frequency pattern state $E$ 
that has been experimentally validated will be tested on models of heterogenous ultra-dense cellular networks for their performance. A practical scenario application of this reconfigurable antenna concerning spatial modulation is presented in [16].

\section{Abbreviations}

5G: 5th generation; F/B: Front-to-back ratio; GHz: Gigahertz;

LPKF: Leiterplatten Kopier Fräsen protomat; MIMO: Multiple-input multipleoutput; NLOS: Non-line-of-sight; PVC: Polyvinyl chloride; QLC: Quasi-lumped couplers; RF: Radio frequency; SLL: Side lobe level; SMA: SubMiniature version A; XPD: Cross-polar discrimination

\section{Authors' contributions}

VB proposed the main idea, performed the CAD modelling, completed the fabrication and measurement experiments, analysed the results and wrote the paper. BB contributed in the array CAD modelling, $180^{\circ}$ phasing network design and pattern measurements. $L C$ and JB read the paper and contributed critical constructive reviews. In addition, JB provided fruitful advice towards improving the antenna element. All authors read and approved the final manuscript.

\section{Funding}

This work was supported by the $5 \mathrm{G}$ wireless project that has received funding from the European Union's Horizon 2020 research and innovation programme under grant agreement no. 641985 and by the University of Cantabria PAR project P111.

\section{Availability of data and materials}

The data used in the analysis and validation of the proposed $4 \times 4$ antenna array was generated by measurements carried out on the fabricated $4 \times 4$ antenna array using the vector network analyser and the anechoic chamber. Though this data has been presented in the form of graphs in the paper, raw data is available on request from the corresponding author.

\section{Competing interests}

The authors declare that they have no competing interests.

Received: 27 April 2019 Accepted: 3 July 2019

Published online: 11 July 2019

\section{References}

1. D. Liu, W. Hong, T.S. Rappaport, C. Luxey, W. Hong, What will 5G antennas and propagation be? IEEE Transactions on Antennas and Propagation 65(12), 6205-6212 (2017)

2. W. Hong et al., Multibeam antenna technologies for $5 \mathrm{G}$ wireless communications. IEEE Transactions on Antennas and Propagation 65(12), 6231-6249 (2017)

3. V. Basavarajappa, B.B. Exposito, L. Cabria, J. Basterrechea, Quasi-opticsinspired low-profile endfire antenna element. EURASIP Journal on Wireless Communications and Networking 2019

4. P.Y. Wang et al., Beam switching antenna based on a reconfigurable cascaded feeding network. IEEE Transactions on Antennas and Propagation 66(2), 627-635 (2018)

5. D. Guan, Y. Zhang, Z. Qian, Y. Li, M. Asaadi, C. Ding, A novel 2-D multibeam antenna without beamforming network. IEEE Transactions on Antennas and Propagation 64(7), 3177-3180 (2016)

6. C.M. Chen, V. Volski, L. Van der Perre, G.A.E. Vandenbosch, S. Pollin, Finite large antenna arrays for massive MIMO: characterization and system impact. IEEE Transactions on Antennas and Propagation 65(12), 6712-6720 (2017)

7. H. Tanabe, K. Sakakibara, N. Kikuma, Multibeam-switching millimeter-wave antenna using beam-tilting design in perpendicular plane to feeding line of microstrip comb-line antenna (2017 IEEE MTT-S International Conference on Microwaves for Intelligent Mobility (ICMIM), Nagoya, 2017), pp. 139-142

8. A.F. Morabito, P. Rocca, Optimal synthesis of sum and difference patterns with arbitrary sidelobes subject to common excitations constraints. IEEE Antennas and Wireless Propagation Letters 9, 623-626 (2010)

9. S.T. Smith, Optimum phase-only adaptive nulling. IEEE Transactions on Signal Processing 47(7), 1835-1843 (1999)
10. A.F. Morabito, A. Massa, P. Rocca, T. Isernia, An effective approach to the synthesis of phase-only reconfigurable linear arrays. IEEE Transactions on Antennas and Propagation 60(8), 3622-3631 (2012)

11. G. Byun, H. Choo, S. Kim, Improvement of pattern null depth and width using a curved array with two subarrays for CRPA systems. IEEE Transactions on Antennas and Propagation 63(6), 2824-2827 (2015)

12. H. Steyskal, R. Shore, R. Haupt, Methods for null control and their effects on the radiation pattern. IEEE Transactions on Antennas and Propagation 34(3), 404-409 (1986)

13. S.A. Schelkunoff, A mathematical theory of linear arrays. The Bell System Technical Journal 22(1), 80-107 (Jan. 1943)

14. V. Basavarajappa, B.B. Exposito, L. Cabria, J. Basterrechea, Millimeter wave multibeam switching antenna (Proc. IEEE ISWCS 2017, Bologna, 2017)

15. D.M. Pozar, A relation between the active input impedance and the active element pattern of a phased array. IEEE Transactions on Antennas and Propagation51(9), 2486-2489 (2003)

16. D.N.Viet, M. D. Renzo, V. Basavarajappa, B. B. Exposito, J. Basterrechea and D. T.P. Huy, Spatial modulation based on reconfigurable antennas:performance evaluation by using the prototype of a reconfigurable antenna. EURASIP Journal on Wireless Communications and Networking 2019 2019:149.

\section{Publisher's Note}

Springer Nature remains neutral with regard to jurisdictional claims in published maps and institutional affiliations.

\section{Submit your manuscript to a SpringerOpen ${ }^{\circ}$ journal and benefit from:}

- Convenient online submission

- Rigorous peer review

- Open access: articles freely available online

High visibility within the field

- Retaining the copyright to your article

Submit your next manuscript at $\boldsymbol{\nabla}$ springeropen.com 